\title{
Valley-polarized tunneling currents in bilayer graphene tunneling transistors
}

\author{
J. J. P. Thompson, ${ }^{1,{ }^{*}}$ D. J. Leech, ${ }^{1,2}$ and M. Mucha-Kruczyński1 ${ }^{1,3}$ \\ ${ }^{1}$ Department of Physics, University of Bath, Claverton Down, Bath BA2 7AY, United Kingdom \\ ${ }^{2}$ Centre for Fine Print Research, University of the West of England Bristol BS3 2JT, United Kingdom \\ ${ }^{3}$ Centre for Nanoscience and Nanotechnology, University of Bath, Claverton Down, Bath BA2 7AY, United Kingdom
}

(Received 13 November 2018; revised manuscript received 20 January 2019; published 15 February 2019)

\begin{abstract}
We study theoretically the electron current across a monolayer graphene/hexagonal boron nitride/bilayer graphene tunneling junction in an external magnetic field perpendicular to the layers. We show that change in effective tunneling barrier width for electrons on different graphene layers of bilayer graphene, coupled with the fact that its Landau level wave functions are not equally distributed amongst the layers with a distribution that is reversed between the two valleys, lead to valley polarization of the tunneling current. We estimate that valley polarization $\sim 70 \%$ can be achieved in high quality devices at $B=1 \mathrm{~T}$. Moreover, we demonstrate that strong valley polarization can be obtained both in the limit of strong-momentum-conserving tunneling and in lower quality devices where this constraint is lifted.
\end{abstract}

DOI: 10.1103/PhysRevB.99.085420

\section{INTRODUCTION}

Electron tunneling through a potential barrier is one of the most widely known physical consequences of quantum mechanics, responsible for effects as varied as nuclear fusion in stars, radioactive decay, or spontaneous DNA mutation [1]. In particular, the probability of successful tunneling decays exponentially with the width of the barrier, an effect best visualized in scanning transmission microscopy where moving the conducting tip away from the sample leads to rapidly decaying tunneling currents, hence allowing for imaging of the corrugation of the sample surface [2]. Recently, the limit of single-atomic-layer barrier thickness has been achieved in van der Waals (vdW) heterostructures using, first, atomically thin graphene and hexagonal boron nitride $(\mathrm{hBN})[3,4]$ as the electrode and barrier, respectively, and later other two-dimensional atomic crystals [5]. The resulting tunneling transistors offer a solution to the graphene "band gap problem"- the lack of a band gap in the conical electronic dispersion of the material [4]. Moreover, in devices with ultrahigh quality interfaces, momentum-conserving tunneling was demonstrated, leading to negative differential resistance [6-11] and valley polarization due to an in-plane magnetic field [12]. It was also shown that electron tunneling in $\mathrm{vdW}$ heterostructures can be accompanied by excitation of various quasiparticles, for example, phonons [13] or magnons [14], and influenced by defects in the tunnel barrier $[15,16]$. Finally, moiré superlattice effects can be used to engineer the electronic densities of states of the electrodes [17-20].

Here, we study theoretically the tunneling current flowing between bilayer graphene (BLG) and monolayer graphene (MLG) electrodes through a hBN barrier, in the presence of a magnetic field perpendicular to the atomic layers. The impact of the applied magnetic field is twofold: First, the electronic

*j.j.p.thompson@bath.ac.uk density of states is modified due to Landau quantisation and, second, layer polarization of the low-energy Landau levels in BLG [21] leads to efficient generation of valley polarization $[22,23]$. We show here that valley polarization of order unity is possible in magnetic fields as low as $\sim 1 \mathrm{~T}$, and that choice of the valley quantum number of the tunneling current can be made electronically without reversing or changing the magnitude of the magnetic field. While the largest valley polarization can be achieved in high quality devices in which tunneling electrons conserve both energy and momentum, our results suggest that even in the absence of momentum conservation, polarization $\sim 70 \%$ can be achieved at $B=1 \mathrm{~T}$.

\section{DEVICE DESCRIPTION AND TUNNELING MATRIX ELEMENT}

The schematic of the device we study is shown in Fig. 1 with the assumed direction of the magnetic field, $B$. We consider BLG, two layers of carbon atoms arranged in regular hexagons and stacked in a Bernal $(A B)$ formation, adjacent to a few-layer hBN sheet with a MLG sheet placed onto the opposite face, such that the hBN acts as a tunneling barrier between the two graphene materials. The BLG and MLG electrodes can be rotationally misaligned so that their crystallographic directions are rotated by an angle $\theta$ [24]. Following experimental device architectures $[8,12,25]$, we assume that both MLG and BLG are encapsulated with hBN. We additionally assume that the misalignment between graphene electrodes and hBN (both the barrier as well as the top and bottom encapsulating layers) is large so that moiré effects like miniband formation [18] or lattice relaxation [26], important for highly aligned interfaces [27], can be neglected. Finally, a silicon back gate which enables doping of the tunneling electrodes is located on the side of MLG, separated by $\mathrm{SiO}_{2}$, while a gold top gate is attached adjacent to the BLG electrode.

To find the tunneling current through the device, we use Bardeen's formalism $[4,9,28-31]$, which utilizes the wave 
B

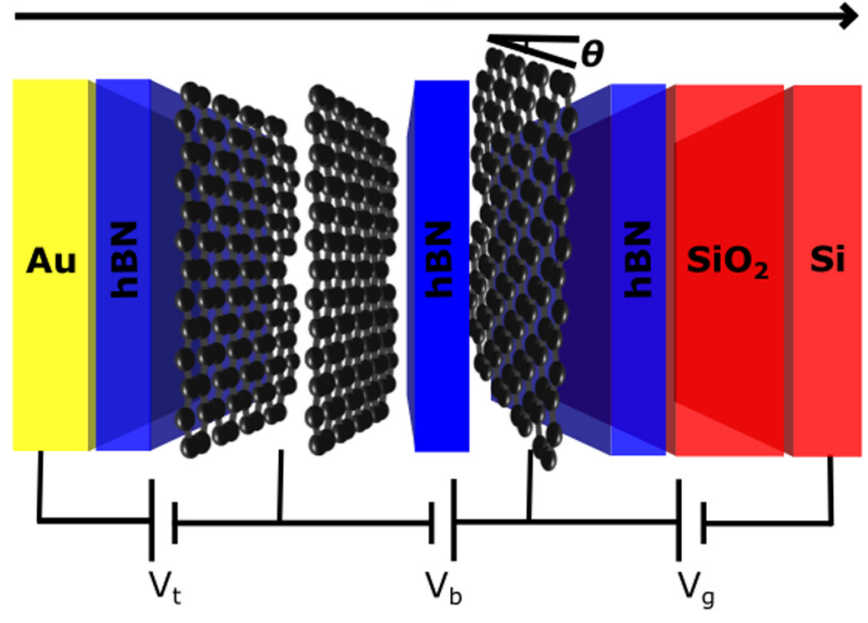

FIG. 1. Schematic of the tunneling device discussed here. Also shown are the tuning potentials $V_{b}, V_{g}$, and $V_{t}$ and direction of the applied magnetic field $B$.

functions of the source and drain electrodes to model the tunneling probability. The matrix element, $M(\varepsilon)$, associated with the probability of an electron with energy $\varepsilon$ tunneling through the barrier (which we take to lie in the $x y$ plane), is calculated (up to some constant prefactor with dimension of energy $\times$ distance) as

$$
M \propto \int\left[\Psi_{\mathrm{S}}(\boldsymbol{r}, \varepsilon) \frac{\partial \Psi_{\mathrm{D}}^{*}(\boldsymbol{r}, \varepsilon)}{\partial z}-\Psi_{\mathrm{D}}(\boldsymbol{r}, \varepsilon) \frac{\partial \Psi_{\mathrm{S}}^{*}(\boldsymbol{r}, \varepsilon)}{\partial z}\right] d V,
$$

where $\Psi_{\mathrm{S}}\left(\Psi_{\mathrm{D}}\right)$ describes the wave function on the source (drain) electrode and the integration is over the volume of the tunneling junction. In a multilayer electrode, such as BLG, the overall wave function can be described as a linear combination of the wave functions on the constituent layers. Therefore, in the case of an $N$-layer source electrode, we can write

$$
\Psi_{\mathrm{S}}(\boldsymbol{r}, \varepsilon)=\sum_{i=1}^{N} n_{i} \psi_{\mathrm{S}, i}(\boldsymbol{r}, \varepsilon),
$$

where $\psi_{\mathrm{S}, i}$ is the electronic wave function of the $i$ th layer of the source electrode and $\left|n_{i}\right|^{2}$ describes the relative occupation of the $i$ th layer by an electron in state $\Psi_{\mathrm{S}}$.

Assuming a clean sample, all $\psi_{\mathrm{S}, i}(\boldsymbol{r}, \varepsilon)$ are separable into the in-plane and perpendicular components, $\psi_{\mathrm{S}, i}(\boldsymbol{r}, \varepsilon)=$ $\varphi_{\mathrm{S}, i}(x, y, \varepsilon) \phi_{S, i}(z, \varepsilon)$. This enables us to decompose the matrix element, Eq. (1), into transverse, $z$, and in-plane, $x, y$, components. We model the transverse component of the wave functions, $\phi_{\mathrm{S}, i}(z, \varepsilon)$, as exponentially decaying, taking care to change the decay constant in the regions corresponding to different materials. In our case, there are two materials to consider: $\mathrm{hBN}$, comprising the tunneling barrier, and graphene. We assign to them decay constants $c(\varepsilon)$ and $c^{\prime}(\varepsilon)$, respectively (the decay constants vary as a function of the tunneling state energy $\varepsilon$ [29]). With these assumptions, we integrate the expression in Eq. (1) in the direction transverse to the barrier. For our BLG source electrode, which consists of two graphene layers, the electrons from the layer closest to the barrier tunnel directly to the drain, passing only through one material, hBN. However, the electrons in the layer further from the barrier have to travel an increased distance. Since there are no available states on the other graphene layer the electrons have to pass through, it can be effectively treated as an insulator and so the only mechanism for transport is tunneling. Integrating over the total width of the barrier for these electrons, defined as the sum of the $\mathrm{hBN}$ barrier width, $d$, and the interlayer separation between graphene layers, $d_{0}$, we obtain an expression depending only on the in-plane components of the wave function and energy,

$$
\begin{aligned}
& M\left(n_{1}, n_{2}, \varepsilon\right) \propto e^{-c(\varepsilon) d}\left[\int \varphi_{\mathrm{D}}^{*}(x, y, \varepsilon) \times\left(\begin{array}{ll}
n_{1} & n_{2} e^{-c^{\prime}(\varepsilon) d_{0}}
\end{array}\right)\right. \\
& \left.\times\left(\begin{array}{l}
\varphi_{\mathrm{S}, 1}(x, y, \varepsilon) \\
\varphi_{\mathrm{S}, 2}(x, y, \varepsilon)
\end{array}\right) d A\right] \text {, }
\end{aligned}
$$

where we have absorbed all normalization factors into $\varphi_{\mathrm{S}, i}(x, y, \varepsilon)$ and used the fact that in our device the drain electrode is built of only one layer and source of two. Note that the expression in Eq. (3) conserves the in-plane electron momentum in the tunneling process, as is the case in experiments performed on the highest quality devices $[6,8,9,12]$.

While the argument above can be extended to any number of layers in both the source and drain electrodes, the exponential dependence of tunneling probability on the barrier width means that only tunneling from/into the first few layers next to the barrier is measurable. For hBN, experimental works $[3,4,9,12,32-34]$ suggest a value of the decay constant $c(0) \approx 5 \mathrm{~nm}^{-1}$. In the case of graphene, studies of its role as a barrier in magnetic tunnel junctions [35-37] and between metal contacts $[38,39]$ showed that it behaves as a strong out-of-plane insulator. In fact, in experiments conducted in the absence of a magnetic field and in the presence of a field parallel to the graphene layers, the measured tunneling current has been well described by assuming that all tunneling from the further BLG layer is suppressed [10,12]. For this reason, here, we take the limit $c^{\prime}=c$, corresponding to the decay through graphene being significant and similar to that through hBN. However, our conclusions hold for notably smaller $c^{\prime}$ (we discuss what happens for differing estimates of $c^{\prime}$ in Appendix A).

\section{WAVE FUNCTIONS OF GRAPHENE ELECTRODES}

To obtain the wave functions of electrons in our BLG and MLG electrodes, we use the low-energy description for electrons in these materials, applicable in the vicinity of the inequivalent Brillouin zone corners (valleys) $\boldsymbol{K}_{\xi}=\xi(4 \pi / 3 a, 0)$, where $\xi= \pm 1$ and the graphene lattice constant $a=2.46 \AA$. A single graphene layer consists of two sublattices, $A$ and $B$, and in the case of BLG, an effective low-energy model can be constructed using Bloch states, $\phi(A 1)$ and $\phi(B 2)$, formed from $p_{z}$-orbitals on the nondimer sites (sites which do not have a neighbor directly above/below them) [21], which we refer to as $A 1$ and $B 2$ with the labels 1 and 2 corresponding to the layer closer and further from the barrier, respectively. For an electron with momentum $\boldsymbol{p}=\left(p_{x}, p_{y}\right)$ measured from the center of valley $\boldsymbol{K}_{\xi}$, the resulting Hamiltonian, written in the 
basis $\{\phi(A 1), \phi(B 2)\}^{T}$ in $\boldsymbol{K}_{+}$and $\{\phi(B 2), \phi(A 1)\}^{T}$ in $\boldsymbol{K}_{-}$, is

$$
\begin{aligned}
\hat{\boldsymbol{H}}_{\mathrm{BLG}} & =-\frac{v^{2}}{\gamma_{1}}\left(\begin{array}{cc}
0 & \pi^{\dagger^{2}} \\
\pi^{2} & 0
\end{array}\right)+\hat{\boldsymbol{H}}_{u}, \\
\hat{\boldsymbol{H}}_{u} & =\frac{\xi u}{2}\left(\begin{array}{cc}
1 & 0 \\
0 & -1
\end{array}\right)+\frac{\xi u v^{2}}{\gamma_{1}^{2}}\left(\begin{array}{cc}
\pi^{\dagger} \pi & 0 \\
0 & \pi \pi^{\dagger}
\end{array}\right), \\
\pi & =p_{x}+i p_{y} .
\end{aligned}
$$

Above, the velocity, $v \approx 10^{6} \mathrm{~ms}^{-1}$, is related to the in-plane nearest-neighbor hopping while $\gamma_{1} \approx 0.38 \mathrm{eV}$ is the vertical interlayer coupling. The term $\hat{\boldsymbol{H}}_{u}$ captures the effect of energy difference between sites on different layers, $u$ (interlayer asymmetry), due to the electric field perpendicular to the BLG electrode induced by the applied voltages.

Misalignment between the MLG and BLG electrodes, generated by a small clockwise rotation of the MLG sheet about the $z$ axis by angle $\theta$, leads to an identical rotation between the corresponding Brillouin zones. As a result of this rotation, the position of MLG valley centers is offset from that of BLG by a vector $\Delta \boldsymbol{K}_{\xi}=\left(\Delta K_{\xi}^{x}, \Delta K_{\xi}^{y}\right)=\left(\hat{\mathbf{1}}-\hat{\boldsymbol{R}}_{\boldsymbol{\theta}}\right) \boldsymbol{K}_{\xi}$, where $\hat{\boldsymbol{R}}_{\boldsymbol{\theta}}$ is the anticlockwise rotation operator. Taking into account this shift as well as the rotation between the two materials, electrons in the MLG electrode are described by a Hamiltonian,

$$
\hat{\boldsymbol{H}}_{\mathrm{MLG}}=v\left(\begin{array}{cc}
0 & \left(\pi^{\dagger}+\tilde{\pi}^{\dagger}\right) e^{-i \theta} \\
(\pi+\tilde{\pi}) e^{i \theta} & 0
\end{array}\right),
$$

which acts on the basis $\{\phi(A), \phi(B)\}^{T}$ for the $\boldsymbol{K}_{+}$valley, and $\{\phi(B),-\phi(A)\}^{T}$ in the $\boldsymbol{K}_{-}$valley, and $\tilde{\pi}=\hbar\left(\Delta K_{\xi}^{x}+i \Delta K_{\xi}^{y}\right)$.

We include the magnetic field $\boldsymbol{B}$ applied perpendicular to the graphene planes in Eqs. (4) and (5) by using the Peierls substitution, $\boldsymbol{p} \rightarrow \boldsymbol{p}+\boldsymbol{e A}$, and the Landau gauge $\boldsymbol{A}=$ $(0,-B x, 0)$. As a result, the operators $\pi$ and $\pi^{\dagger}$ become lowering and raising operators, respectively, for functions built of quantum harmonic oscillator states along the $x$ direction, $\phi_{m}(x)$, and plane waves along the $y$ direction,

$$
\begin{aligned}
\pi \phi_{m}(x) e^{i k_{y} y} & =-i \frac{2 \hbar}{\lambda_{B}} \sqrt{m} \phi_{m-1}(x) e^{i k_{y} y}, \\
\pi^{\dagger} \phi_{m}(x) e^{i k_{y} y} & =i \frac{2 \hbar}{\lambda_{B}} \sqrt{m+1} \phi_{m+1}(x) e^{i k_{y} y} \\
\phi_{m}(x) & =A_{m} \exp \left[-\frac{1}{2 \lambda_{B}^{2}}(x-X)^{2}\right] \mathcal{H}_{m}\left[\frac{1}{\lambda_{B}}(x-X)\right] \\
A_{m} & =\frac{1}{\sqrt{2^{m} m ! \sqrt{\pi} \lambda_{B} L}},
\end{aligned}
$$

where $\lambda_{B}=\sqrt{\hbar / e B}$ is the magnetic length, $\mathcal{H}_{m}(x)$ is a Hermite polynomial of order $m, L$ is the dimension of the flake along the $y$ direction and $X=\lambda_{B}^{2} k_{y}$ is the position of the center of cyclotron orbit of an electron with wave vector $k_{y}$. Using Eqs. (6), the energies of the BLG Landau levels, can be expressed as [21]

$$
\begin{aligned}
\varepsilon_{0, \xi} & =\frac{\xi u}{2}, \quad \varepsilon_{1, \xi}=\frac{\xi u}{2}-\xi \eta^{2} u, \\
\varepsilon_{m, s, \xi} & =-\frac{\eta^{2} \xi u}{2}+s \sqrt{\left(\varepsilon_{m}^{0}\right)^{2}+\frac{1}{4} u^{2}}, \quad m \geqslant 2,
\end{aligned}
$$

where $\eta=\sqrt{2} v \hbar / \lambda_{B} \gamma_{1}, \varepsilon_{m}^{0}=\gamma_{1} \eta^{2} \sqrt{m(m-1)}$ and $s= \pm 1$ is the conduction/valence band index in BLG, defined when $m \geqslant$ 2 . The corresponding wave functions are

$$
\begin{aligned}
\psi_{0} & =\left(\begin{array}{c}
\phi_{0} \\
0
\end{array}\right) e^{i k_{y} y}, \quad \psi_{1}=\left(\begin{array}{c}
\phi_{1} \\
0
\end{array}\right) e^{i k_{y} y}, \\
\psi_{m, s}^{\xi} & =\frac{1}{\sqrt{2 C}}\left(\begin{array}{c}
C_{1} \phi_{m} \\
C_{2} \phi_{m-2}
\end{array}\right) e^{i k_{y} y}, \quad m \geqslant 2, \\
C_{1} & =\varepsilon_{m}^{0}, \quad C_{2}=\left[\varepsilon_{m, s, \xi}-\xi \frac{u}{2}+\xi u m \eta^{2}\right],
\end{aligned}
$$

where $C=C_{1}^{2}+C_{2}^{2}$ is the normalization constant.

In rotated MLG, the displacement of the momentum-origin modifies the harmonic oscillator state, $\tilde{\phi}_{n}(x)$,

$$
\begin{aligned}
\tilde{\phi}_{n}(x)= & A_{n} \exp \left[-\frac{1}{2 \lambda_{B}^{2}}(x-\tilde{X})^{2}-i \Delta K_{\xi}^{x}(x-\tilde{X})\right] \\
& \times \mathcal{H}_{n}\left[\frac{1}{\lambda_{B}}(x-\tilde{X})\right],
\end{aligned}
$$

and shifts the cyclotron orbits to $\tilde{X}=\lambda_{B}^{2}\left(k_{y}+\Delta K_{\xi}^{y}\right)$. The resulting energy levels in MLG are

$$
\varepsilon_{0}=0, \quad \varepsilon_{n, s^{\prime}}=s^{\prime}\left(\sqrt{2} v \hbar / \lambda_{B}\right) \sqrt{n}, \quad n \geqslant 1,
$$

where $s^{\prime}= \pm 1$ is the conduction/valence band index in MLG, defined when $n \geqslant 1$. Therefore, the wave function corresponding to the $n$th Landau level in rotated MLG can be written as

$$
\tilde{\psi}_{0}=\left(\begin{array}{c}
\tilde{\phi}_{0} \\
0
\end{array}\right) e^{i k_{y} y}, \quad \tilde{\psi}_{n, s^{\prime}}=\frac{1}{\sqrt{2}}\left(\begin{array}{c}
\tilde{\phi}_{n} \\
-s^{\prime} i e^{i \theta} \tilde{\phi}_{n-1}
\end{array}\right) e^{i k_{y} \cdot y}, \quad n \geqslant 1 .
$$

In MLG, all the Landau levels have an additional fourfold degeneracy due to spin and valley. Moreover, the $n=0$ Landau level is positioned at the Dirac point and its energy does not depend on the magnetic field. In BLG, for $u=0$, in addition to the valley and spin degeneracies of each level, both the $m=0$ and $m=1$ Landau levels sit at the neutrality point, leading to an unusual eightfold degenerate zero-energy state. Nonzero $u$ lifts both the $m=0,1$ and valley degeneracies.

In both MLG and BLG, the wave functions are distributed asymmetrically between the two sublattices concerned ( $A, B$ in MLG and $A 1, B 2$ in BLG). In particular, the electrons in the $n=0$ MLG level and $m=0,1$ BLG states occupy only one of the sublattices [40]. For the case of BLG, this results in two states that in the $\boldsymbol{K}_{+}$valley are located only on layer 1 and in the $\boldsymbol{K}_{-}$valley only on layer 2 . In the vertical tunneling transistor geometry like in Fig. 1, we expect electrons from layer 2 to have a smaller chance of tunneling through the barrier than electrons from layer 1 , due to the additional effective barrier thickness. As a result, more electrons from BLG $\boldsymbol{K}_{+}$valley will tunnel through than from the $\boldsymbol{K}_{-}$valley, leading to valley-polarized current arriving in the MLG drain electrode.

To quantify valley polarization of the tunneling current, we use the wave functions from Eqs. (8) and (11) to compute the tunneling matrix element, Eq. (3) (see Appendix B for more details). The Landau-level wave functions of BLG are 
already written so that their components correspond to different layers and we can identify the states in Eqs. (8) with $\left(\varphi_{\mathrm{S}, 1}(x, y, \varepsilon), \varphi_{\mathrm{S}, 2}(x, y, \varepsilon)\right)^{T}$ in Eq. (3). However, although the MLG wave functions are also written as spinors in Eq. (11), both of their components correspond to wave-function amplitudes on sublattices in the same layer. Hence, for a given Landau-level state, we take $\varphi_{D}=\chi_{A}+\chi_{B}$, where $\left(\chi_{A}, \chi_{B}\right)^{T}$ is the corresponding spinor in Eq. (11).

In clean samples, with a small misalignment angle between the source and drain electrodes, the shift $\boldsymbol{\Delta} \boldsymbol{K}_{\xi}$ is small and for all the dominant processes the valley quantum number is conserved in the tunneling process $[9,12]$. Therefore, we use Fermi's golden rule to relate the tunneling matrix element to current of electrons originating in the $\boldsymbol{K}_{\xi}$ valley of BLG,

$$
\begin{aligned}
I_{\xi}= & \frac{4 \pi e}{\hbar} \sum_{n, m, s^{\prime}, s} \int_{\mu_{\mathrm{BLG}}}^{\mu_{\mathrm{MLG}}+\Delta}\left|M_{n, m, \xi}^{s, s^{\prime}}(\varepsilon)\right|^{2} \\
& \times D_{\mathrm{BLG}}\left(\varepsilon, \varepsilon_{m, s, \xi}\right) D_{\mathrm{MLG}}\left(\varepsilon-\Delta, \varepsilon_{n, s}\right) d \varepsilon,
\end{aligned}
$$

where we have already taken the spin degeneracy into account. We measure the energy $\varepsilon$ from the charge neutrality point of the BLG electrode while $\mu_{\mathrm{BLG}}$ and $\mu_{\mathrm{MLG}}$ represent the distance in energy between the charge neutrality point and the chemical potential in the BLG and MLG electrodes, respectively. Finally, we define $\Delta$ as the shift between the source and drain neutrality points such that, in the low-temperature limit, the local chemical potentials in the source and drain electrodes, $\mu_{\mathrm{BLG}}$ and $\mu_{\mathrm{MLG}}+\Delta$, respectively, determine the energy window within which tunneling processes can occur, while the number of initial and final states at a given energy is provided by the densities of states $D_{\mathrm{MLG}}$ and $D_{\mathrm{BLG}}$ in the monolayer and BLG, respectively. In a device with high quality layers, free from defects and in a quantizing external magnetic field, these densities of states consist of a series of sharp peaks at the energies of the Landau levels. We model the latter using a Lorentzian shape with the same full width at half maximum for all Landau levels, $2 \mathrm{meV}$ and $4 \mathrm{meV}$ for $B=1$ $\mathrm{T}$ and $B=4 \mathrm{~T}$, respectively, following previous experimental works $[9,41]$ and theoretical considerations [42]. Finally, we define the valley polarization, $P$, of the tunneling current,

$$
P=\frac{I_{+}-I_{-}}{I_{+}+I_{-}}
$$

Because our tunneling matrix element, Eq. (1), is defined up to a proportionality constant, values of tunneling current in this paper are given in arbitrary units. Polarization, however, as a ratio of currents, does not depend on that constant itself.

We set the thickness of the hBN barrier (separation between the MLG and BLG) to $d=13 \AA$ and the interlayer distance in BLG as $d_{0}=3.3 \AA$. We also relate the energies $\varepsilon, \mu_{\mathrm{BLG}}$, and $\mu_{\mathrm{MLG}}$ to the applied voltages $V_{t}, V_{b}$, and $V_{g}$ (see Fig. 1) through the electrostatic equations,

$$
\begin{aligned}
V_{b} & =\frac{1}{e}\left[\mu_{\mathrm{BLG}}-\mu_{\mathrm{MLG}}-\Delta\right], \\
V_{g} & =-\frac{e\left(n_{\mathrm{MLG}}+n_{\mathrm{BLG}}+n_{\mathrm{Au}}\right)\left(d_{\mathrm{SiO}_{2}} \epsilon_{\mathrm{hBN}}+d_{\mathrm{hBN}} \epsilon_{\mathrm{SiO}_{2}}\right)}{\epsilon_{\mathrm{hBN}} \epsilon_{\mathrm{SiO}_{2}} \epsilon_{0}}, \\
V_{t} & =\frac{-e n_{\mathrm{Au}} d_{\mathrm{Top}}}{\epsilon_{0} \epsilon_{\mathrm{hBN}}}
\end{aligned}
$$

discussed in more detail in Appendix C. We define $n_{\mathrm{MLG}}, n_{\mathrm{BLG}}$, and $n_{\mathrm{Au}}$ as the carrier densities on the MLG, BLG, and gold electrodes, respectively. The distance between the gold top gate and BLG, $d_{\text {Top }}$, is set as $30 \mathrm{~nm}$ in our numerical calculations. Furthermore, $d_{\mathrm{hBN}}$ and $d_{\mathrm{SiO}_{2}}$ represent the thicknesses of the $\mathrm{hBN}$ and $\mathrm{SiO}_{2}$ substrates, which, following previous experimental works, we set as $30 \mathrm{~nm}$ and $300 \mathrm{~nm}$, respectively. Finally, $\epsilon_{0}$ is the permittivity of free space while $\epsilon_{\mathrm{hBN}} \approx 3$ and $\epsilon_{\mathrm{SiO}_{2}} \approx 3.9$ are the relative permittivities of $\mathrm{hBN}$ and $\mathrm{SiO}_{2}$. We also take into account that the electric field between the graphene layers of BLG induces the interlayer asymmetry $u$, which we compute self-consistently,

$$
u=-\frac{e^{2} d_{0}\left(n_{\mathrm{Au}}+n_{\mathrm{BLG}, 2}(u)\right)}{\epsilon_{0}},
$$

where $n_{\mathrm{BLG}, i}(u)$ is the carrier density on the $i$ th layer of BLG. For a given interlayer asymmetry, we compute the electronic wave functions for all Landau levels included in the calculation and their distributions on the atomic sites (while the number of Landau levels considered depended on the magnetic field and applied voltage range, we checked the convergence of our results in all cases). For each Landau level, we use the square of the wave function amplitude on the site $B 2$ to obtain the contribution to $n_{\mathrm{BLG}, 2}$ from that level. We then determine the unique value of $u$ for which Eq. (15) is fulfilled.

\section{MOMENTUM-CONSERVING TUNNELING}

\section{A. Total tunneling current at $B=1 \mathrm{~T}$}

Our simulation of the total tunneling current, $I=I_{+}+$ $I_{-}$, between the BLG and MLG electrodes, produced using Eq. (12), is shown in Fig. 2. We indicate the boundaries of regions corresponding to fixed lowest filling factors $v_{\mathrm{MLG}}$ and $v_{\mathrm{BLG}}$ in MLG and BLG, respectively, with the grey lines and label these regions as $\left(v_{\mathrm{MLG}}, \nu_{\mathrm{BLG}}\right)$ [43]. For momentumconserving tunneling, the strength of the coupling is dictated by the magnitude of the applied magnetic field, relative orientation of the electrodes, and Landau-level indices of the involved electronic states.

In Fig. 2(a), we show the current for $V_{t}=0 \mathrm{~V}$ and ideally aligned electrodes, $\theta=0^{\circ}$. For $V_{g}=V_{b}=V_{t}=0 \mathrm{~V}$, the chemical potentials in the BLG and MLG electrodes, $\mu_{\mathrm{BLG}}$ and $\mu_{\mathrm{MLG}}+\Delta$, are located at their respective neutrality points, which are at the same energy, resulting in zero tunneling current. As the bias voltage is increased, a shift between the local chemical potentials in the two electrodes is induced. This opens an energy window, within which electrons occupying states in one electrode can tunnel into empty states at the same energy in the other electrode, thus leading to a nonzero tunneling current. The coupling strength between the initial and final states in the tunneling process is set by $\left|M_{n, m, \xi}^{s, s^{\prime}}(\varepsilon)\right|^{2}$ which, because of the spinorial nature of the MLG and BLG wave functions, is expressed as a sum of four terms, each of which contains an integral $\int \tilde{\phi}_{n}^{*} \phi_{m} d A$ of two oscillator states $\tilde{\phi}_{n}$ and $\phi_{m}$. For ideal alignment of the electrodes, $\theta=0^{\circ}$, the set $\left\{\tilde{\phi}_{n}\right\}$ is equivalent to $\left\{\phi_{m}\right\}$ and the integrals express orthonormality of functions with different indices, $\int \phi_{n}^{*} \phi_{m} d A=\delta_{n, m}$. As a result, tunneling only occurs if one of the four conditions is fulfilled: (i) $n=m$, 
(a)

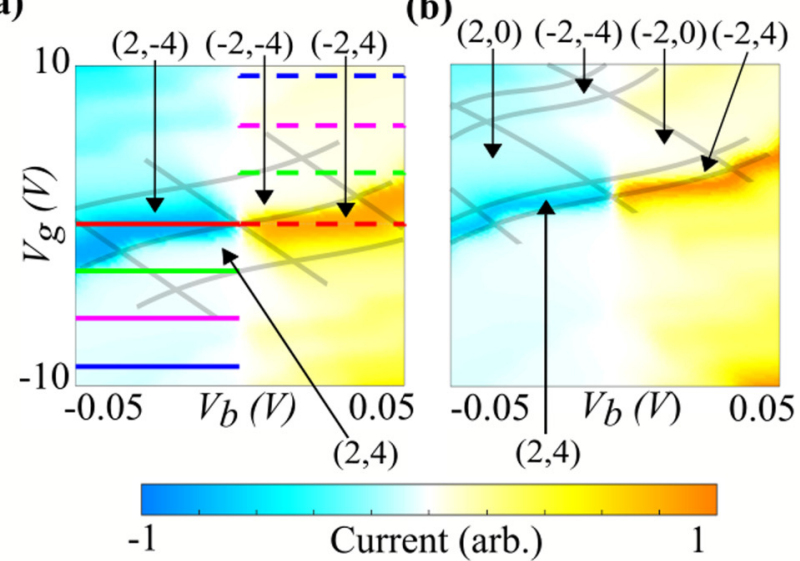

(c)

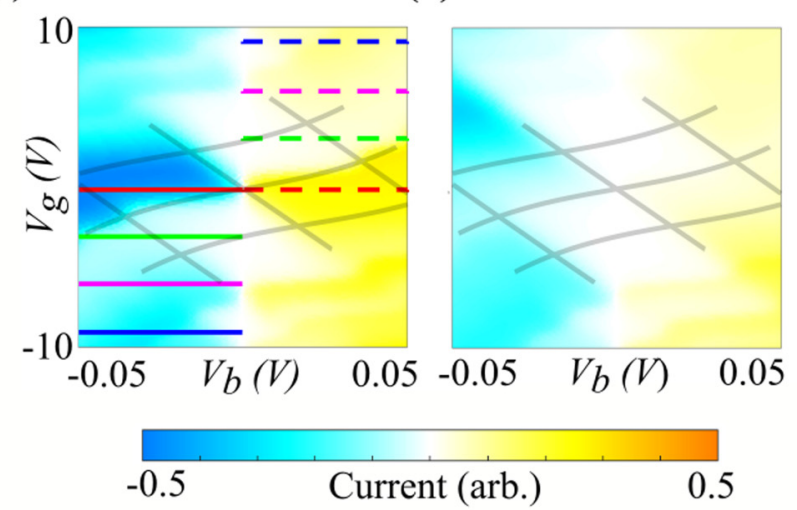

(e)

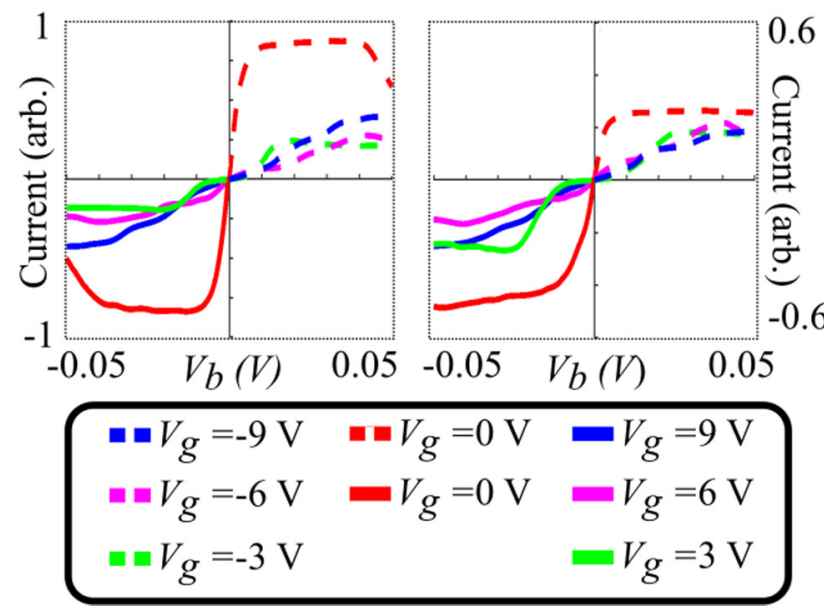

FIG. 2. Total tunneling current at $B=1 \mathrm{~T}$ as a function of the bias and gate voltages, $V_{b}$ and $V_{g}$, and for (a) $\theta=0^{\circ}$ and $V_{t}=0 \mathrm{~V}$, (b) $\theta=0^{\circ}$ and $V_{t}=0.5 \mathrm{~V}$, (c) $\theta=0.25^{\circ}$ and $V_{t}=0 \mathrm{~V}$, and (d) $\theta=0.5^{\circ}$ and $V_{t}=0 \mathrm{~V}$. Grey lines in (a) and (b) indicate the boundaries of regions of constant filling factors labeled as $\left(v_{\mathrm{MLG}}, v_{\mathrm{BLG}}\right)$ with the first (second) filling factor corresponding to monolayer (bilayer) graphene. For clarity, we do not show labels of these regions in panels (c) and (d) for which they are the same as in (a). Panels (e) and (f) show current curves corresponding to the lines marked in (a) and (c), respectively, with changing $V_{b}$ and constant $V_{g}$ from $-9 \mathrm{~V}$ to $9 \mathrm{~V}$ in steps of $3 \mathrm{~V}$. (ii) $n-1=m$, (iii) $n=m-2$, and (iv) $n-1=m-2$. Hence, the central region of large current in Fig. 2(a), for $V_{g}=0$ and nonzero $V_{b}$ [fingerlike features across the regions $\left(\mu_{\mathrm{MLG}}, \mu_{\mathrm{BLG}}\right)=(2,-4)$ and $\left.(-2,4)\right]$, corresponds to the coupling between $m=0$ and $n=0$ Landau levels in BLG and MLG, respectively. Although at low voltages the $m=0$ and $m=1$ Landau levels in BLG are degenerate, transitions between the $m=1$ and $n=0$ level in MLG are forbidden. Moreover, although increasing $V_{b}$ increases the size of the tunneling energy window to include higher Landau levels, due to the selection rules for $\theta=0$, these do not contribute to the tunneling current.

Setting nonzero $V_{g}$ at constant $V_{b}$ dopes the graphene electrodes, shifting the two chemical potentials together such that the difference between them remains unchanged. At small $V_{b}$ and zero $V_{g}$ in Fig. 2(a), clear current is observed. However, as $V_{g}$ is increased (decreased), the electrodes become holedoped (electron-doped) and the filling factors are changed to $(-2,-4)[(2,4)]$. As a result, the tunneling energy window, set by $V_{b}$, moves away from the positions of the $m=0$ and $n=0$ Landau levels and the current decreases. Additionally, $V_{b}$ and $V_{g}$ induce an electric field between the graphene layers in BLG which leads to nonzero interlayer asymmetry $u$. This opens a band gap in the electronic spectrum of BLG [21] and hence affects the current characteristics of the device. Within the voltage window shown in Fig. 2(a), $u$ is the largest in the top-right/bottom-left corners of the $\left(V_{b}, V_{g}\right)$ diagram and reaches the magnitude of $\sim 20 \mathrm{meV}$.

Finally, the current diagram as shown in Fig. 2(a) seems to have inversion antisymmetry with respect to the point $\left(V_{b}, V_{g}\right)=(0,0), I\left(V_{b}, V_{g}\right)=-I\left(-V_{b},-V_{g}\right)$. In fact, within the voltage window presented in Fig. 2, this antisymmetry is only weakly broken by the energy dependence of the decay coefficient $c(\varepsilon)$ (see also Appendix A)-a feature also observed experimentally $[3,4]$. We investigate this symmetry in more detail in Fig. 2(e) where we present current plots for changing $V_{b}$ and constant $V_{g}$ from $-9 \mathrm{~V}$ to $9 \mathrm{~V}$ in steps of 3 $\mathrm{V}$ corresponding to solid/dashed lines marked in Fig. 2(a). We show with solid lines current for negative $V_{g}$ and $V_{b}$ and, with dashed lines, current for positive $V_{b}$ and $V_{g}$. The same color is used for curves with the same magnitude of $V_{g}$ and, for all $V_{g}$, we have $I\left(V_{g}, V_{b}\right)$ almost equal to $-I\left(-V_{g},-V_{b}\right)$.

In Fig. 2(b), we show the tunneling current as a function of $V_{b}$ and $V_{g}$ for $V_{t}=0.5 \mathrm{~V}$. Nonzero $V_{t}$ induces interlayer asymmetry, $u$, even for $V_{b}=V_{g}=0 \mathrm{~V}$ while also introducing a shift between the MLG and BLG neutrality points, $\Delta$. The former leads to valley splitting, resulting in new filling factor regions with $\nu_{\mathrm{BLG}}=0$, while the latter leads to energy misalignment of the $m=n=0$ Landau levels. However, because in BLG the position of the $m=0$ Landau level depends linearly on $u$ [see Eqs. (7)], the impact of $V_{t}$ can be counterbalanced by choosing $V_{g}$ such that the overall $u$ shifts the $m=0$ BLG Landau level in the $K_{+}$valley back into alignment with the $n=0$ Landau level in MLG. This restores the fingerlike feature in Fig. 2(b) visible for some positive $V_{g}$.

In Figs. 2(c) and 2(d), we show the impact of misalignment $\theta=0.25^{\circ}$ and $\theta=0.5^{\circ}$, respectively, between the two 
electrodes on the tunneling current. For nonzero $\theta$, the oscillator functions $\tilde{\phi}_{n}$ and $\phi_{m}$ are no longer orthonormal and transitions between any pair of states are allowed. Moreover, as shown in Appendix B, the coupling strength between states also depends on the misalignment angle. For these reasons, in Figs. 2(c) and 2(d), the fingerlike feature present in Figs. 2(a) and 2(b) becomes increasingly smeared out with increasing $\theta$ and the tunneling current also decreases as compared to Figs. 2(a) and 2(b). Additionally, misalignment between the electrodes breaks the approximate inversion antisymmetry of the current diagram in Fig. 2(a). In the presence of $\theta \neq 0^{\circ}$, each of the four terms of the kind $\int \tilde{\phi}_{n} \phi_{m} d A$ appearing in the calculation of the matrix element $M_{n, m, \xi}^{s, s^{\prime}}(\varepsilon)$ (see also Appendix B) comes with a prefactor that depends on the MLG and BLG band indices $s^{\prime}$ and $s$. Upon inversion from $I\left(V_{g}, V_{b}\right)$ to $I\left(-V_{g},-V_{b}\right)$, the interference between these terms leads to different results, depending on whether the initial and final states originate in the conduction or valence band. As a consequence, the approximate inversion antisymmetry about $\left(V_{g}, V_{b}\right)=(0,0)$, present in Fig. 2(a) is strongly broken in both Figs. 2(c) and 2(d). This is demonstrated in more detail in Fig. 2(f), where we show similar current curves as in Fig. 2(e) (changing $V_{b}$ for constant $V_{g}$ from $-9 \mathrm{~V}$ to $9 \mathrm{~V}$ in steps of $3 \mathrm{~V}$ with the same color scheme) produced for $\theta=0.25^{\circ}$ [the cuts are also indicated in Fig. 2(c)]. In particular, the magnitude of the current for $V_{g}=0 \mathrm{~V}$ is much larger for $V_{b}<0 \mathrm{~V}$ than $V_{b}>0 \mathrm{~V}$.

\section{B. Valley polarization at $B=1 \mathrm{~T}$}

As the Landau-level wave functions of BLG are not distributed equally between its two constituent graphene layers and this distribution is reversed between the valleys, tunneling in the device shown in Fig. 1 can be used to produce unequal electron occupations in the MLG drain electrode. Such an effect can be characterized by the valley polarization, $P$, of the tunneling current, introduced in Eq. (13), which we plot in Fig. 3 as a function of the gate voltages $V_{g}$ and $V_{b}$ for $B=1$ T. Figures $1(a)-1(d)$ correspond to the same parameters for which we presented total tunneling current in Figs. 2(a)-2(d).

In Fig. 2(a), we show the case of $\theta=0^{\circ}$ and $V_{t}=0 \mathrm{~V}$. The polarization diagram has inversion symmetry with respect to $\left(V_{b}, V_{g}\right)=(0,0)$. A bright red crosslike feature corresponds to $P \sim 50 \%$ with a region of $P \sim 80 \%$ in the center of the diagram. This high valley polarization is due to the tunneling between the $m=0$ BLG and $n=0$ MLG Landau levels. In the BLG $\boldsymbol{K}_{+}$valley, electrons in the $m=0$ state occupy exclusively the layer closer to the barrier, whereas in the $\boldsymbol{K}_{-}$ valley all of them sit on the layer further from the barrier. Consequently, the current in the $\boldsymbol{K}_{+}$valley is significantly larger than in the $\boldsymbol{K}_{-}$valley.

As $V_{g}$ is increased, the $m=0$ and $n=0$ Landau levels move out of alignment and the dominant source of tunneling current becomes the $m=2$ to $n=0$ transition. From Eqs. (8), the BLG $m=2$ wave function is $\psi_{2, s}^{\xi} \propto\left(C_{1} \phi_{2}, C_{2} \phi_{0}\right)^{T}$, where $C_{1}$ and $C_{2}$ are complex numbers, and the first and second components of $\psi_{2, s}^{\xi}$ are located, respectively, on layer 1 (layer 2) and layer 2 (layer 1 ) in valley $\boldsymbol{K}_{+}\left(\boldsymbol{K}_{-}\right)$. For the MLG $n=0, \tilde{\psi}=\left(\tilde{\phi_{0}}, 0\right)$, so that for $\theta=0^{\circ}$, tunneling in $\boldsymbol{K}_{+}$is only possible for BLG electrons from layer 2, further from (a)

(b)

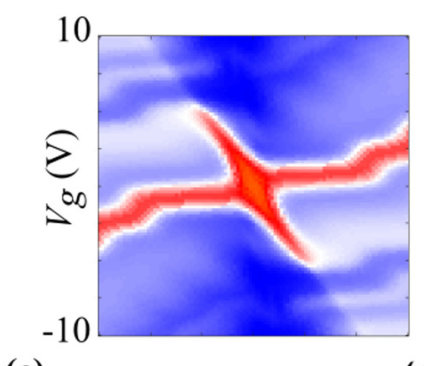

(c)

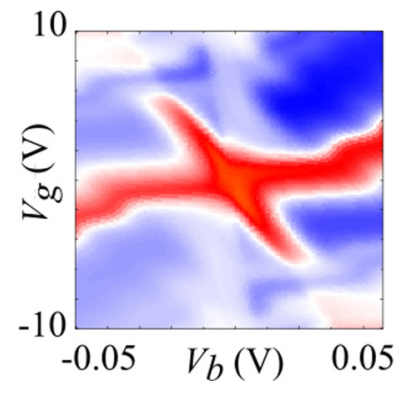

(d)

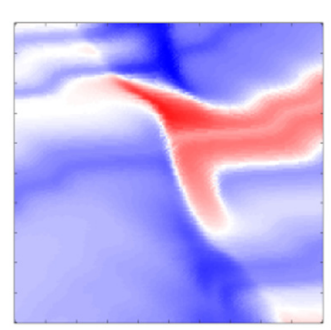

$\mathrm{K}+$
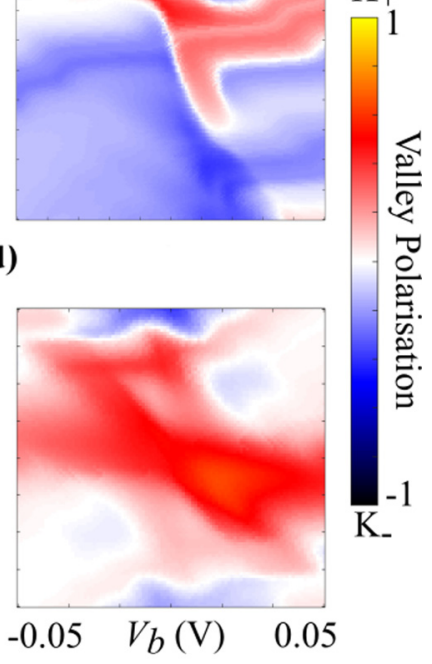

FIG. 3. Valley polarization of the tunneling current at $B=1 \mathrm{~T}$, as a function of the bias and gate voltages $V_{b}$ and $V_{g}$. Each of the panels (a)-(d) corresponds to the total current shown in the equivalent panels (a)-(d) of Fig. 2: (a) $\theta=0^{\circ}$ and $V_{t}=0 \mathrm{~V}$, (b) $\theta=0^{\circ}$ and $V_{t}=0.5 \mathrm{~V}$, (c) $\theta=0.25^{\circ}$ and $V_{t}=0 \mathrm{~V}$, and (d) $\theta=0.5^{\circ}$ and $V_{t}=0$ V. Positive (negative) polarization indicates current favoring the $\boldsymbol{K}_{+}$ $\left(\boldsymbol{K}_{-}\right)$valley.

the barrier, while in $\boldsymbol{K}_{-}$it is the electrons from layer 1 that can tunnel into MLG. As a result, the overall current has negative $\left(\boldsymbol{K}_{-}\right)$polarization as shown by dark blue regions above and below the central red cross in Fig. 3(a). Similar arguments can be used to explain other regions of the polarization diagram.

For nonzero top-gate voltage, as shown in Fig. 3(b) for $V_{t}=0.5 \mathrm{~V}$, the polarization map is modified as a result of the shift between the neutrality points, $\Delta$, as well as nonzero interlayer asymmetry, $u$, at $\left(V_{b}, V_{g}\right)=(0,0)$. The latter lifts the valley degeneracy of the BLG $m=0$ Landau level. Alignment of the $\boldsymbol{K}_{+}$BLG $m=0$ and MLG $n=0$ states, responsible for the red crosslike feature in Fig. 3(a), now requires compensating with positive gate voltage. However, for negative $V_{b}$ it is not possible to both align these two states and position the BLG and MLG chemical potentials such that the aligned states contribute to the current. As a result, the left arm of the red cross disappears and the $m=2$ to $n=0$ transitions lead to negative polarization in this region.

In Figs. 3(c) and 3(d), we show valley polarization as a function of $V_{b}$ and $V_{g}$ for increasing misalignment between the electrodes, $\theta=0.25^{\circ}$ and $\theta=0.5^{\circ}$, corresponding to total current plots in Figs. 2(c) and 2(d). Similar to the current features, when the graphene electrodes are misaligned, individual polarization features become smeared out and the variation of polarization throughout the $\left(V_{b}, V_{g}\right)$-space becomes more gradual. The oscillator states $\phi_{m}$ and $\tilde{\phi}_{n}$ are not orthonormal for $\theta \neq 0^{\circ}$, so that many different transitions contribute to the overall polarization for given $\left(V_{b}, V_{g}\right)$. Importantly, interference of electronic states tunneling between any of the BLG layers and any of the MLG sublattices which leads to different 
(a)

(b)

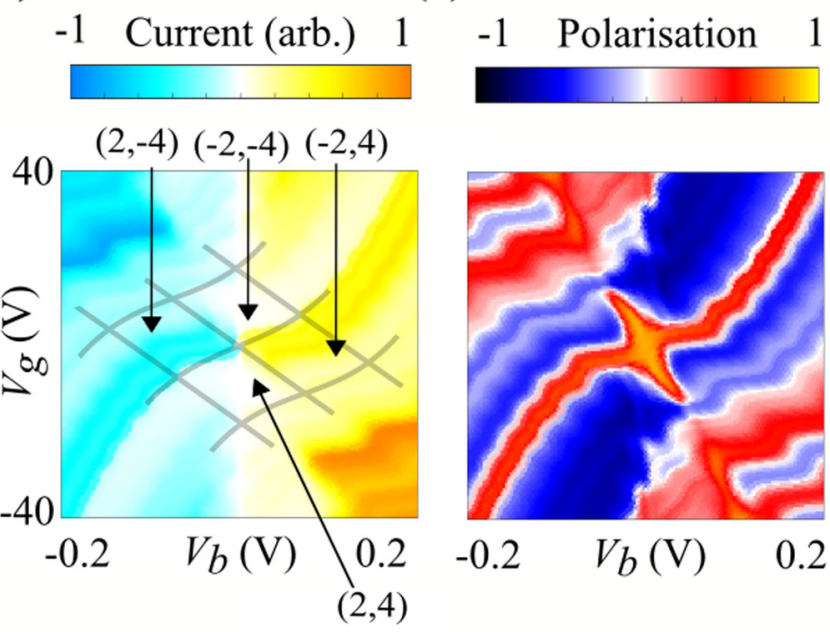

FIG. 4. Total tunneling current (a) and valley polarization (b) as a function of the bias and gate voltages $V_{b}$ and $V_{g}$ for magnetic field $B=4 \mathrm{~T}, \theta=0$, and $V_{t}=0 \mathrm{~V}$. The maximum polarization observed is as much as $90 \%$ in favor of the $\boldsymbol{K}_{+}$valley. Grey lines [in (a)] indicate the boundaries of regions of constant filling factors labeled $\left(v_{\mathrm{MLG}}, v_{\mathrm{BLG}}\right)$, with the first (second) filling factor corresponding to monolayer (bilayer) graphene. The filling factor regions in (b) are identical to those in (a).

outcomes for conduction band-conduction band and valence band-valence band transitions, strongly breaks the inversion symmetry of polarization present in Fig. 3(a) for $\theta=0^{\circ}$. This symmetry breaking grows with increasing $\theta$.

\section{Tunneling at $B=4 \mathrm{~T}$}

The Landau-level structures in BLG and MLG depend on the strength of the magnetic field differently, hence the tunneling current and polarization features in the $\left(V_{b}, V_{g}\right)$ diagrams depend on $B$. For this reason, to contrast our results for $B=1 \mathrm{~T}$ presented in Figs. 2 and 3 with the case of stronger magnetic field, in Fig. 4 we show the tunneling current and its valley polarization for $B=4 \mathrm{~T}, \theta=0^{\circ}$, and $V_{t}=0 \mathrm{~V}$. Due to the increased electron density per Landau level at $B=4 \mathrm{~T}$, it is necessary to increase the voltage range to compare features arising from similar electronic tunneling transitions. Similar to the $B=1 \mathrm{~T}$ case, a fingerlike structure is present across the regions $\left(\mu_{\mathrm{MLG}}, \mu_{\mathrm{BLG}}\right)=(2,-4)$ and $(-2,4)$ in Fig. 4(a). In fact, it is more pronounced because the separation between the $m=n=0$ Landau levels and the rest of the electronic spectra in the corresponding materials is increased. Consequently, the central crosslike region of $\boldsymbol{K}_{+}$-polarized current is also sharper, including polarization of $P \sim 90 \%$ in the vicinity of $\left(V_{b}, V_{g}\right)=(0,0)$. The maximum $\boldsymbol{K}_{-}$polarization in the blue region dominated by $m=2$ to $n=0$ tunneling is also increased.

\section{TUNNELING WITH STRONG MOMENTUM SCATTERING}

In the presence of a poor interface between the electrodes and the hBN barrier, the scattering length-scale becomes very (a)

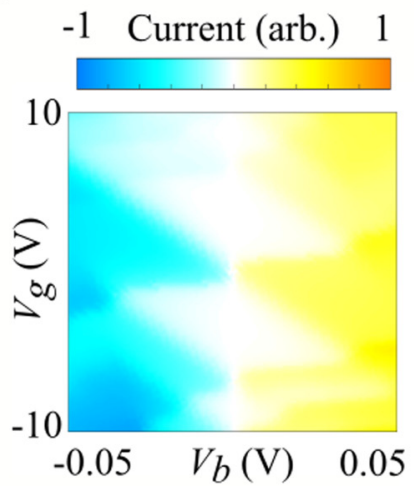

(b)

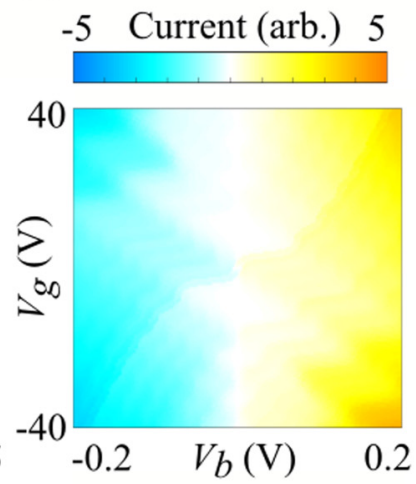

(c)

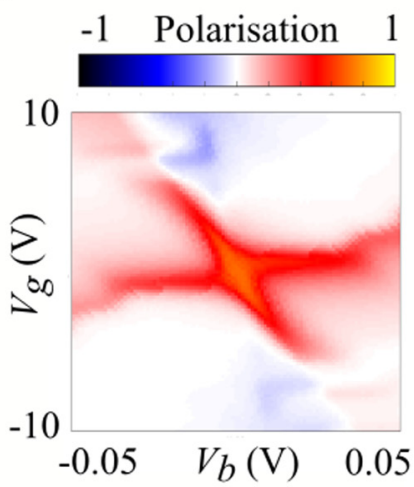

(d)

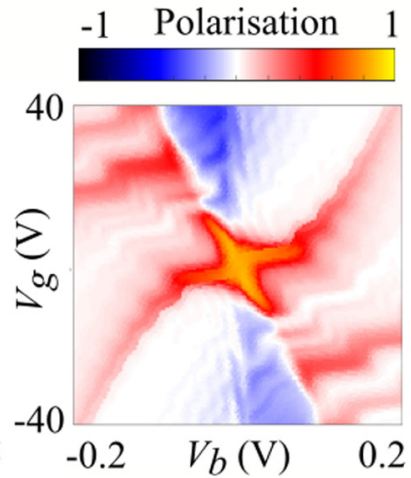

FIG. 5. Electron transport through the proposed device in the absence of momentum conservation and as a function of the bias and gate voltages $V_{b}$ and $V_{g}$ with $V_{t}=0$. Panels (a) and (b) show the total tunneling current for $\theta=0^{\circ}$, (a) $B=1 \mathrm{~T}$, and (b) $B=4 \mathrm{~T}$. Panels (c) and (d) present valley polarization of the current shown in (a) and (b), respectively.

small such that the momentum resolution of the tunneling electron becomes lost. In this limit, the momentum nature of the initial and final state has no effect on the magnitude and valley polarization of the current across the device. Instead, the tunneling current depends only on the density of states of the source and drain electrode. As a consequence, we expect the valley polarization of the tunneling current to arise purely due to differences in valley occupations of the two BLG layers. We model this regime by setting each harmonic oscillator integral, $\int \tilde{\phi}_{n} \phi_{m} d A$, equal to 1 for all the transitions independently of their initial and final states [44] and present our results for $\theta=0^{\circ}$ and $V_{t}=0 \mathrm{~V}$ in Fig. 5 .

In panels (a) and (b) of Fig. 5, we show the tunneling current for $B=1 \mathrm{~T}$ and $B=4 \mathrm{~T}$, respectively. Because all of the transitions are now allowed, the graphs look similar to that in Fig. 2(d), corresponding to $\theta=0.5^{\circ}$. In Fig. 2(b), the increased magnitude of the magnetic field leads to larger spacing between the Landau levels so that, as compared to Fig. 2(a), a larger voltage window is necessary to capture features due to transitions between the same pair of Landau levels.

In Figs. 5(c) and 5(d), we present valley polarization of the currents shown in Figs. 5(a) and 5(b), respectively. The relaxation of the selection rules discussed in the previous 
section results in polarization maps which are heavily weighted in favor of $K_{+}$valley. In particular, the maximum valley polarization occurs at low voltages, where the participating Landau levels are those with low index (in particular $m=0$ and $m=1$ ). This is because, for the $m=0$ and $m=1$ BLG Landau levels, the valley and layer degrees of freedom are coupled. Furthermore, the interlayer asymmetry, $u$, generates a layer population difference in the $m \geqslant 2$ Landau levels in BLG, which is opposite in the two valleys. This induced interlayer asymmetry is responsible for small regions of minor $K_{-}$polarization which occur at higher voltages. These two principles are responsible for all polarization features observed in Figs. 5(c) and 5(d).

The relative misalignment of the graphene electrodes has no effect on the tunneling probability in this limit. Similar to the case of momentum-conserving tunneling between misaligned electrodes, $\theta \neq 0^{\circ}$, lack of restrictions on allowed transitions leads to chiral interference. This interference results in the asymmetry in inversion about the origin that is observed in Fig. 5. We expect momentum nonconserving tunneling to be the dominant mechanism when the misalignment angle between the graphene electrodes is large. This is because, while increasing misalignment angle decreases the magnitude of the momentum-conserving current, it should have no effect on the transitions involving scattering.

\section{SUMMARY}

We have explored the tunneling characteristics of a vertical field-effect transistor comprising monolayer and BLG electrodes, in the presence of a perpendicular magnetic field. The coupled layer and valley polarization in the Landau levels of BLG gives rise to a valley-polarized tunneling current through the device, resulting in unequal valley populations in MLG. Our result is due to the difference in effective tunneling barrier widths for electrons in the two layers of the BLG electrode. As such, valley polarization should persist in the presence of small local variations of the tunneling rates (and hence effective tunneling decay lengths). Importantly, this valley polarization can be tuned solely by electrostatic means without the need to reverse the direction of the magnetic field. Our modeling suggests that $P \sim \pm 70 \%$ is possible in high quality devices in homogeneous fields of $B=1 \mathrm{~T}$. Fields of such magnitude could be, in principle, generated by placing ferromagnets on top of the device [45]. While the homogeneity of the field distribution across the device would then depend on the size of the ferromagnet, thickness of the tunneling junction, and distance between the two, valley polarization might still be possible in such a setup.

In both the momentum-conserving and nonconserving regimes, the most persistent feature in valley polarization plots is the crosslike region of $\boldsymbol{K}_{\mathbf{+}}$-polarized current around $\left(V_{g}, V_{b}\right)=(0,0)$. In the same voltage region, the total tunneling current forms a fingerlike pattern. Both originate in tunneling current from $m=0(m=0,1$ in the absence of momentum conservation) to $n=0$, so that observing the fingerlike features in the current should indicate a region of considerable valley polarization. To detect the valley polarization produced using the proposed device directly, two stacks could be connected in series: the first one to produce unequal valley populations and the second to act as a detector. Alternatively, the produced valley polarization can be measured using optical means [46].

\section{ACKNOWLEDGMENTS}

The authors acknowledge helpful discussions with E. McCann and K. Takashina. This work has been supported by the EPSRC through the University of Bath Doctoral Training Partnership, Grants No. EP/M50645X/1 and No. EP/M507982/1.

\section{APPENDIX A: CHANGING THE DECAY CONSTANT OF GRAPHENE}

In the main text, we take the decay constant $c^{\prime}(\varepsilon)$ characterizing tunneling through MLG to be equivalent to the decay constant $c(\varepsilon)$ corresponding to tunneling through $\mathrm{hBN}$. Here we discuss the effect of changing the decay constant $c^{\prime}(\varepsilon)$, on our results. Following previous work [4,47], we relate the decay constant to the height of the tunneling barrier. For the case of hBN, we treat it as an isotropic potential step with barrier height $\Phi_{0}=-1.5 \mathrm{eV}$, corresponding to experimental measurements of the valence band maximum (VBM) of hBN $[4,5]$. The $\mathrm{hBN}$ energy dispersion around the VBM is roughly parabolic in $k_{z}$ and this allows us to write [47]

$$
c(\varepsilon)=\operatorname{Im} \frac{\sqrt{2 m^{*} \Phi(\varepsilon)}}{\hbar}=\operatorname{Im} \frac{\sqrt{2 m^{*}\left(\Phi_{0}-\varepsilon\right)}}{\hbar},
$$

where $m^{*}$ is the effective mass. The above relation predicts weak electron-hole asymmetry in tunneling current (as observed in experiments [4,5]). In our paper, we use the expression in Eq. (A1) to obtain the decay constant $c^{\prime}(\varepsilon)=c(\varepsilon)$ for the tunneling of BLG electrons from the layer further from the barrier across the graphene layer closer to the barrier. While both the barrier height and the effective mass would be different for graphene as compared to hBN (here, for the sake of the numerical calculations, for hBN we take $m^{*}=$ $0.5 m_{0}$, following previous modeling of vertical tunneling in graphene/hBN stacks [4,5]), our main conclusions are quite insensitive to the numerical values of $c^{\prime}(\varepsilon)$ and $c(\varepsilon)$. In fact, the latter impacts both electrons tunneling from the top and bottom BLG layers in the same way and hence leads to an identical numerical coefficient for all tunneling processes for given applied voltages. The physics we describe arises primarily due to the additional exponential factor, $\exp \left(-c^{\prime}(\varepsilon) d_{0}\right)$, in tunneling from the bottom layer as compared to the top one.

In Fig. 6, we demonstrate the valley polarization at $B=1$ $\mathrm{T}$ and $\theta=0^{\circ}$ for effective graphene decay constant, $c^{\prime}(\varepsilon)$, scaled by a factor of (a) $\frac{1}{\sqrt{2}}$ and (b) $\frac{1}{2}$ as compared to the $\mathrm{hBN}$ value provided by Eq. (A1). While the asymmetry between $P\left(V_{b}, V_{g}\right)$ and $P\left(-V_{b},-V_{g}\right)$ increases slightly for smaller decay constant, qualitative features of the valley polarization graphs remain the same. Also, the maximum valley polarizations are still significant, $58 \%$ and $48 \%$, respectively, compared to $70 \%$ in Fig. 3(a). Increasing $c^{\prime}(\varepsilon)$ (making graphene more insulating) increases the valley polarization of the tunneling current. 
(a)

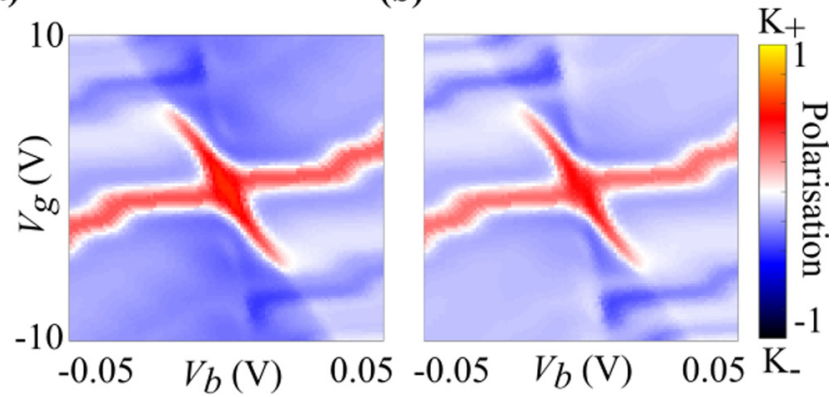

FIG. 6. Valley polarization for momentum-conserving tunneling between perfectly aligned electrodes $\left(\theta=0^{\circ}\right)$ and for $B=1 \mathrm{~T}$ as a function of $V_{b}$ and $V_{g}$ with $V_{t}=0 \mathrm{~V}$. In contrast to the polarization shown in Fig. 3(a), the plots here are obtained using the decay constant decreased by a factor (a) $\frac{1}{\sqrt{2}}$ and (b) $\frac{1}{2}$ (compared to in the main text).

\section{APPENDIX B: LANDAU-LEVEL COUPLINGS AND MATRIX ELEMENT IN THE MOMENTUM-CONSERVING LIMIT}

The matrix element determining the tunneling between the BLG and MLG electrodes depends on the Landau-level indices, $n, m$, as well as the magnetic field, $B$, and misalignment angle between the two sheets, $\theta$. For the $\boldsymbol{K}_{+}$valley, it can be written as

$$
\begin{aligned}
M_{n, m, K_{+}}^{s^{\prime}, s}(\varepsilon)= & \frac{V_{0} P_{n, m}}{\sqrt{2}} e^{-c(\varepsilon) d} \times\left[C_{1} \Lambda_{n, m}^{\xi}-s^{\prime} i e^{i \theta} C_{1} \Lambda_{n-1, m}^{\xi}\right. \\
& \left.+e^{-c^{\prime}(\varepsilon) d_{0}}\left(C_{2} \Lambda_{n, m-2}^{\xi}-s^{\prime} i e^{i \theta} C_{2} \Lambda_{n-1, m-2}^{\xi}\right)\right],
\end{aligned}
$$

whereas, for the $\boldsymbol{K}_{-}$valley, we obtain

$$
\begin{aligned}
M_{n, m, K_{-}}^{s^{\prime}, s}(\varepsilon)= & \frac{V_{0} P_{n, m}}{\sqrt{2}} e^{-c(\varepsilon) d}\left[C_{2} \Lambda_{n,|m|-2}^{\xi}+s^{\prime} i e^{-i \theta} C_{2} \Lambda_{n-1, m-2}^{\xi}\right. \\
& \left.+e^{-c^{\prime}(\varepsilon) d_{0}}\left(C_{1} \Lambda_{n, m}^{\xi}+s^{\prime} i e^{-i \theta} C_{1} \Lambda_{n-1, m}^{\xi}\right)\right] . \quad \text { (B2) }
\end{aligned}
$$

In both cases, we define

$$
\begin{aligned}
C_{1}= & \begin{cases}\frac{\varepsilon_{m}^{0}}{\sqrt{C}} & m \neq 0,1 \\
1 & m=0,1\end{cases} \\
C_{2}= & \begin{cases}\frac{\left[\varepsilon_{m, s, \xi}-\xi \frac{u}{2}+\xi u m \eta^{2}\right]}{\sqrt{C}} & m \neq 0,1, \\
0 & m=0,1\end{cases} \\
\Lambda_{n, m}^{\xi}= & N_{n, m} 2^{\max \{n, m\}}(\min \{n, m\}) ! e^{i \frac{1}{2} \Delta K_{\xi}^{x} \Delta K_{\xi}^{y} \lambda_{B}^{2}} \\
& \left.\times\left(\operatorname{sgn}(n-m) \frac{1}{2} \lambda_{B} \Delta K_{\xi}^{y}-i \frac{1}{2} \lambda_{B} \Delta K_{\xi}^{x}\right)\right)^{|n-m|} \\
& \times e^{-\frac{\Delta K_{\xi}^{2} \lambda_{B}^{2}}{4}} \mathcal{L}_{\min \{n, m\}}^{|n-m|}\left(\frac{\Delta K_{\xi}^{2} \lambda_{B}^{2}}{2}\right),
\end{aligned}
$$

where $N_{n, m}$ and $P_{n, m}=\sqrt{\left(1+\delta_{n, 0}\right)}$ are normalization constants and $\mathcal{L}_{\alpha}^{\beta}(x)$ are generalized Laguerre polynomials. The strength of the coupling at the $\boldsymbol{K}_{+}$valley, $\left|\boldsymbol{M}_{n, m, K_{+}}^{s^{\prime}, s}\right|^{2}$, is shown for $B=1 \mathrm{~T}$ and as a function of Landau level, $n, m$, and band (a)

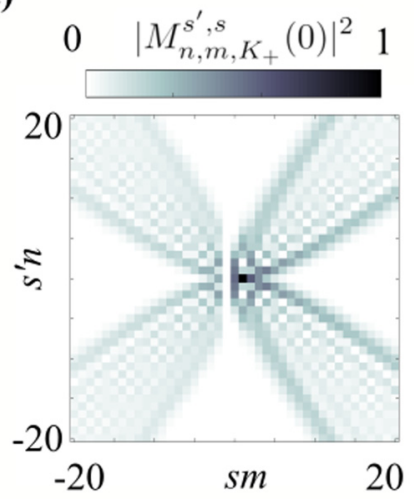

(b)

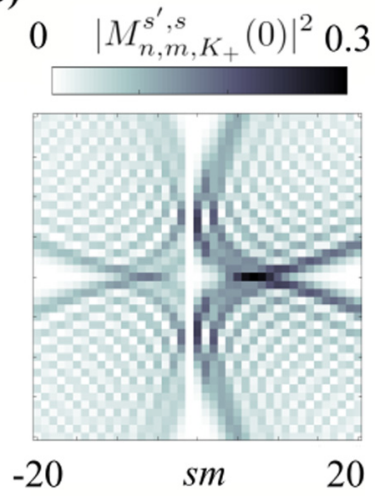

FIG. 7. Color map of the tunneling matrix element $\left|M_{n, m, K_{+}}^{s, s^{\prime}}(0)\right|^{2}$ between Landau levels of MLG (with indices $s^{\prime} n$ ) and gapless BLG (indices $s m$ ) for $B=1 \mathrm{~T}$, (a) $\theta=0.25^{\circ}$, and (b) $\theta=0.5^{\circ}$. All values are normalized to the maximum value in (a).

indices, $s, s^{\prime}$, in Figs. 7(a) and 7(b) at misalignment angles $\theta=0.25^{\circ}$ and $\theta=0.5^{\circ}$, respectively.

For zero misalignment angle, the matrix element is simply a linear combination of Kronecker deltas (each expressing orthonormality of the harmonic oscillator states), suggesting only transitions between certain Landau states are allowed. However, as shown in Fig. 7(a), increasing misalignment redistributes the coupling strength amongst other transitions (in particular, for any nonzero $\theta$, transitions between any two oscillator states are, in principle, allowed). Interestingly, changing the misalignment angle also changes the preferred transition (the one with the largest coupling strength). However, because the matrix element depends on products of the type $\Delta \boldsymbol{K}_{\xi}^{2} \lambda_{B}^{2}$, a change of angle (which determines $\Delta \boldsymbol{K}_{\xi}$ ) can be to some extent counterbalanced by changing the magnetic field (and hence $\lambda_{B}$ ).

\section{APPENDIX C: ELECTROSTATICS}

The bias voltage $V_{b}$ and gate voltages $V_{g}$ and $V_{t}$ control the local Fermi levels in BLG and MLG as well as the shift between the neutrality points and the interlayer asymmetry in BLG. We use a four-plate capacitor model to express the electric fields between the gates and consecutive graphene layers (we treat $\mathrm{hBN}$ and $\mathrm{SiO}_{2}$ as homogeneous insulators with dielectric constants $\epsilon_{\mathrm{hBN}}$ and $\epsilon_{\mathrm{SiO}_{2}}$, respectively). The carrier densities per graphene layer on the BLG source $(j=$ BLG) or MLG drain ( $j=$ MLG) electrodes can be expressed as

$$
\begin{aligned}
n_{j}= & \frac{1}{\pi^{2} \lambda_{B}^{2}} \sum_{m, s, \xi}\left[\arctan \left(\frac{\mu_{j}-\varepsilon_{m, s}^{\xi}}{\Gamma_{j}}\right)\right. \\
& \left.-\arctan \left(\frac{-\varepsilon_{m, s}^{\xi}}{\Gamma_{j}}\right)\right] .
\end{aligned}
$$

Through charge conservation, for each combination of $\mu_{\mathrm{BLG}}$, $\mu_{\mathrm{MLG}}$, and $u$, we obtain corresponding bias, bottom and top gate potentials. Furthermore, the charge buildup on the bilayer sheet acts as a capacitance leading to a difference in neutrality 
points of the two spectra:

$$
\Delta=E_{0}^{\mathrm{MLG}}-E_{0}^{\mathrm{BLG}} .
$$

For simplicity, we set $E_{0}^{\mathrm{BLG}}=0$ and therefore the position of the charge neutrality point in the monolayer is related to the number of excess charge carriers in the BLG by relation

$$
E_{0}^{\mathrm{MLG}}=\Delta=-\frac{e^{2}\left(n_{\mathrm{Au}}+n_{\mathrm{BLG}}\right) d}{\epsilon_{0} \epsilon_{\mathrm{hBN}}} .
$$

The voltages of the system can therefore be expressed as

$$
\begin{aligned}
V_{b} & =\frac{1}{e}\left[\mu_{\mathrm{BLG}}-\mu_{\mathrm{MLG}}-\Delta\right], \\
V_{g} & =-\frac{e\left(n_{\mathrm{MLG}}+n_{\mathrm{BLG}}+n_{\mathrm{Au}}\right)\left(d_{\mathrm{SiO}_{2}} \epsilon_{\mathrm{hBN}}+d_{\mathrm{hBN}} \epsilon_{\mathrm{SiO}_{2}}\right)}{\epsilon_{\mathrm{hBN}} \epsilon_{\mathrm{SiO}_{2}} \epsilon_{0}}, \\
V_{t} & =\frac{-e n_{\mathrm{Au}} d_{\mathrm{Top}}}{\epsilon_{0} \epsilon_{\mathrm{hBN}}} .
\end{aligned}
$$

[1] F. Trixler, Quantum tunneling to the origin and evolution of life, Curr. Org. Chem. 17, 1758 (2013).

[2] G. Binnig, H. Rohrer, Ch. Gerber, and E. Weibel, Surface Studies by Scanning Tunneling Microscopy, Phys. Rev. Lett. 49, 57 (1982).

[3] L. Britnell, R. V. Gorbachev, R. Jalil, B. D. Belle, F. Schedin, M. I. Katsnelson, L. Eaves, S. V. Morozov, A. S. Mayorov, N. M. R. Peres, A. H. Castro Neto, J. Leist, A. K. Geim, L. A. Ponomarenko, and K. S. Novoselov, Electron tunneling through ultrathin boron nitride crystalline barriers, Nano Lett. 12, 1707 (2012).

[4] L. Britnell, R. V. Gorbachev, R. Jalil, B. D. Belle, F. Schedin, A. Mishchenko, T. Georgiou, M. I. Katnelson, L. Eaves, S. V. Morozov, N. M. R. Peres, J. Leist, A. K. Geim, K. S. Novoselov, and L. A. Ponomarenko, Field-effect tunneling transistor based on vertical graphene heterostructures, Science 335, 947 (2012).

[5] T. Georgiou, R. Jalil, B. D. Belle, L. Britnell, R. V. Gorbachev, S. V. Morozov, Y. J. Kim, A. Gholinia, S. J. Haigh, O. Makarovsky, L. Eaves, L. A. Ponomarenko, A. K. Geim, K. S. Novoselov, and A. Mishchenko, Vertical field-effect transistor based on graphene- $\mathrm{WS}_{2}$ heterostructures for flexible and transparent electronics, Nat. Nano. 8, 100 (2013).

[6] L. Britnell, R. V. Gorbachev, A. K. Geim, L. A. Ponomarenko, A. Mishchenko, M. T. Greenaway, T. M. Fromhold, K. S. Novoselov, and L. Eaves, Resonant tunneling and negative differential conductance in graphene transistors, Nat. Comms. 4, 1794 (2013).

[7] S. Kang, Bilayer graphene-hexagonal boron nitride heterostructure negative differential resistance interlayer tunnel FET, IEEE Electron Device Lett. 36, 405 (2015).

[8] A. Mishchenko, J. S. Tu, Y. Cao, R. V. Gorbachev, J. R. Wallbank, M. T. Greenaway, V. E. Morozov, S. V. Morozov, M. J. Zhu, S. L. Wong, F. Withers, C. R. Woods, Y. J. Kim, K. Watanabe, T. Taniguchi, E. E. Vdovin, O. Makarovsky, T. M. Fromhold, V. I. Fal'ko, A. K. Geim, L. Eaves, and K. S. Novoselov, Twist-controlled resonant tunneling in graphene/boron nitride/graphene heterostructures, Nat. Nano. 9, 808 (2014).

[9] M. T. Greenaway, E. E. Vdovin, A. Mishchenko, O. Makarovsky, A. Patan, J. R. Wallbank, Y. Cao, A. V. Kretinin, M. J. Zhu, S. V. Morozov, V. I. Fal'ko, K. S. Novoselov, A. K. Geim, T. M. Fromhold, and L. Eaves, Resonant tunneling between the chiral Landau states of twisted graphene lattices, Nat. Phys. 11, 1057 (2015).

[10] T. L. M. Lane, J. R. Wallbank, and V. I. Fal'ko, Twist-controlled resonant tunneling between monolayer and bilayer graphene, Appl. Phys. Lett. 107, 203506 (2015).
[11] B. Fallahazad, K. Lee, S. Kang, J. M. Xue, S. Larentis, C. Corbet, K. Kim, H. C. P. Movva, T. Taniguchi, K. Watanabe, L. F. Register, S. K. Banarjee, and E. Tutuc, Gate-tunable resonant tunneling in double bilayer graphene heterostructures, Nano Lett. 15, 428 (2015).

[12] J. R. Wallbank, D. Ghazaryan, A. Misra, Y. Cao, J. S. Tu, B. A. Piot, M. Potemski, S. Pezzini, S. Wiedmann, U. Zietler, T. L. M. Lane, S. V. Morozov, M. T. Greenaway, L. Eaves, A. K. Geim, V. I. Fal'ko, K. S. Novoselov, and A. Mishchenko, Tuning the valley and chiral quantum state of dirac electrons in van der Waals heterostructures, Science 353, 575 (2016).

[13] E. E. Vdovin, A. Mishchenko, M. T. Greenaway, M. J. Zhu, D. Ghazaryan, A. Misra, Y. Cao, S. V. Morozov, O. Makarovsky, T. M. Fromhold, A. Patanè, G. J. Slotman, M. I. Katsnelson, A. K. Geim, K. S. Novoselov, and L. Eaves, Phonon-Assisted Resonant Tunneling of Electrons in Graphene-Boron Nitride Transistors, Phys. Rev. Lett. 116, 186603 (2016)

[14] D. Ghazaryan, M. T. Greenaway, Z. Wang, V. H. GuarochicoMoreira, I. J. Vera-Marun, J. Yin, Y. Liao, S. V. Morozov, O. Kristanovski, A. I. Lichtenstein, M. I. Katsnelson, F. Withers, A. Mishchenko, L. Eaves, A. K. Geim, K. S. Novoselov, and A. Misra, Magnon-assisted tunneling in van der Waals heterostructures based on $\mathrm{CrBr}_{3}$, Nat. Electron. 1, 344 (2018).

[15] U. Chandni, K. Watanabe, T. Taniguchi, and J. P. Eisenstein, Signatures of phonon and defect-assisted tunneling in planar metal-hexagonal boron nitride-graphene junctions, Nano Lett. 16, 7982 (2016).

[16] U. Chandni, K. Watanabe, T. Taniguchi, and J. P. Eisenstein, Evidence for defect-mediated tunneling in hexagonal boron nitride-based junctions, Nano Lett. 15, 7329 (2015).

[17] G. H. Li, A. Luican, J. M. B. L. dos Santos, A. H. Castro Neto, A. Reina, J. Kong, and E. Y. Andrei, Observation of Van Hove singularities in twisted graphene layers, Nat. Phys. 6, 109 (2010).

[18] M. Yankowitz, J. M. Xue, D. Cormode, J. D. SanchazYamagishi, K. Watanabe, T. Taniguchi, P. Jarillo-Herrero, P. Jacquod, and B. J. LeRoy, Emergence of superlattice Dirac points in graphene on hexagonal boron nitride, Nat. Phys. 8, 382 (2012).

[19] D. J. Leech, J. J. P. Thompson, and M. Mucha-Kruczyński, Negative Differential Resistance in van der Waals Heterostructures Due to Moiré-Induced Spectral Reconstruction, Phys. Rev. Appl. 10, 034014 (2018).

[20] A. Summerfield, A. Kozikov, T. S. Cheng, A. Davies, Y. Cho, A. N. Khlobystov, C. J. Mellor, C. Thomas Foxon, K. Watanabe, T. Taniguchi, L. Eaves, K. S. Novoselov, S. V. Novikov, and P. H. Beton, Moiré-modulated conductance of hexagonal boron nitride tunnel barriers, Nano Lett. 18, 4241 (2018) 
[21] E. McCann and V. I. Fal'ko, Landau-Level Degeneracy and Quantum Hall Effect in a Graphite Bilayer, Phys. Rev. Lett. 96, 086805 (2006).

[22] A. Rycerz, J. Tworzydło, and C. W. J. Beenakker, Valley filter and valley valve in graphene, Nat. Phys. 3, 172 (2007)

[23] J. R. Schaibley, H. Yu, G. Clark, P. Rivera, J. S. Ross, K. L. Seyler, W. Yao, and X. Xu, Valleytronics in 2D materials, Nat. Rev. Mat. 1, 16055 (2016)

[24] K. Kim, M. Yankowitz, B. Fallahazad, S. Kang, H. C. P. Movva, S. Q. Huang, S. Larentis, C. M. Corbet, T. Taniguchi, K. Watanabe, S. K. Banerjee, B. J. LeRoy, and E. Tutuc, Van der Waals heterostructures with high accuracy rotational alignment, Nano Lett. 16, 1989 (2016).

[25] C. R. Dean, A. F. Young, I. Meric, C. Lee, L. Wang, S. Sorgenfrei, K. Watanabe, T. Taniguchi, P. Kim, K. L. Shepard, and J. Hone, Boron nitride substrates for high-quality graphene electronics, Nat. Nano. 5, 722 (2010).

[26] C. R. Woods, L. Britnell, A. Eckmann, R. S. Ma, J. C. Lu, H. M. Guo, X. Lin, G. L. Yu, Y. Cao, R. V. Gorbachev, A. V. Kretinin, J. Park, L. A. Ponomarenko, M. I. Katsnelson, Yu. N. Gornostyrev, K. Watanabe, T. Taniguchi, C. Casiraghi, H.-J. Gao, A. K. Geim and K. S. Novoselov, Commensurateincommensurate transition in graphene on hexagonal boron nitride, Nat. Phys. 10, 451 (2014)

[27] J. Jung, E. Laksono, A. M. DaSilva, A. H. MacDonald, M. Mucha-Kruczyński, and S. Adam, Moiré band model and band gaps of graphene on hexagonal boron nitride, Phys. Rev. B 96, 085442 (2017)

[28] J. Bardeen, Tunnelling From a Many-Particle Point of View, Phys. Rev. Lett. 6, 57 (1961).

[29] J. Simmons, Generalized formula for the electric tunnel effect between similar electrodes separated by a thin insulating film, J. Appl. Phys. 34, 1793 (1963).

[30] M. Li, D. Esseni, G. Snider, D. Jena, and H. Grace Xing, Single particle transport in two-dimensional heterojunction interlayer tunneling field effect transistor, J. Appl. Phys. 115, 074508 (2014).

[31] R. M. Feenstra, D. Jena, and G. Gu, Single-particle tunneling in doped graphene-insulator-graphene junctions, J. Appl. Phys. 111, 043711 (2012).

[32] T. Roy, L. Liu, S. de la Barrera, B. Chakrabarti, Z. R. Hesab1, C. A. Joiner, R. M. Feenstra, G. Gu, and E. M. Vogel, Tunnelling characteristics in chemical vapor deposited graphene-hexagonal boron nitride-graphene junctions, Appl. Phys. Lett. 104, 123506 (2014).

[33] G. H. Lee, Y. J. Yu, C. Lee, C. Dean, K. L. Shepard, P. Kim, and J. Hone, Electron tunneling through atomically flat and ultrathin hexagonal boron nitride, Appl. Phys. Lett. 99, 243114 (2011).

[34] S. Ge, K. M. Masum Habib, A. De, Y. Barlas, D. Wickramaratne, M. R. Neupane, and R. K. Lake, Interlayer transport through a graphene/rotated boron nitride/graphene heterostructure, Phys. Rev. B 95, 045303 (2017).

[35] A. L. Friedman, O. M. J. van't Erve, C. H. Li, J. T. Robinson, and B. T. Jonker, Homoepitaxial tunnel barriers with functionalized graphene-on-graphene for charge and spin transport, Nat. Comm. 5, 3161 (2014).

[36] A. L. Friedman, O. M. J. van't Erve, J. T. Robinson, K. E. Whitener Jr., and B. T. Jonker, Homoepitaxial graphene tunnel barriers for spin transport, AIP Adv. 6, 056301 (2016).

[37] O. M. J. van 't Erve, A. L. Friedman, E. Cobas, C. H. Li, J. T. Robinson, and B. T. Jonker, Low-resistance spin injection into silicon using graphene tunnel barriers, Nat. Nano. 7, 737 (2012).

[38] M. A. Kuroda, J. Tersoff, D. M. Newns, and G. J. Martyna, Conductance through Multilayer Graphene Films, Nano Lett. 11, 3629 (2011).

[39] X. Zhong, R. G. Amorim, A. R. Rocha, and R. Pandey, Hybridization effects on the out-of-plane electron tunneling properties of monolayers: Is h-BN more conductive than graphene?, Nanotechnology 25, 345703 (2014).

[40] The single-sublattice occupation of the $n=1$ BLG Landau level is not preserved beyond the low-energy two-band model we use here [48]. However, it remains a good approximation for the magnetic fields considered here.

[41] T. Taychatanapat, K. Watanabe, T. Taniguchi and P. JarilloHerrero, Quantum Hall effect and Landau-level crossing of Dirac fermions in trilayer graphene, Nat. Phys. 7, 621 (2011).

[42] H. Funk, A. Knorr, F. Wendler, and E. Malic, Microscopic view on Landau level broadening mechanisms in graphene, Phys. Rev. B. 92, 205428 (2015).

[43] Because at $B=1 \mathrm{~T}$, the Landau-level separation in BLG, $\eta^{2} \gamma_{1} \approx 3.5 \mathrm{meV}$, is comparable to our Landau-level broadening of $2 \mathrm{meV}$, we draw the boundaries between regions by simply rounding the electron density to the nearest filling factor.

[44] This limit is equivalent to setting the scattering length much smaller than the magnetic length $\left(\sigma \ll \lambda_{B}\right)$ in the theoretical model employed in Ref. [9].

[45] A. Nogaret, Electron dynamics in inhomogeneous magnetic fields, J. Phys. Condens. Matter 22, 253201 (2010).

[46] F. Hipolito, and V. M. Pereira, Second harmonic spectroscopy to optically detect valley polarization in 2D materials, 2D Mater. 4, 021027 (2017).

[47] J. G. Simmons, Formula for the electric tunnel effect between similar electrodes separated by a thin insulating film, J. App. Phys. 34, 6 (1963).

[48] M. Mucha-Kruczyński, D. Abergel, E. McCann, and V. Fal'ko, On spectral properties of bilayer graphene: The effect of an $\mathrm{SiC}$ substrate and infrared magneto-spectroscopy, J. Phys. Condens. Matter 21, 344206 (2009). 